\title{
Benchmarking of the JEFF-3.1 library against critical integral experiments
}

\author{
I. Duhamel ${ }^{\mathrm{a}}$ and S. Evo
}

Institute of Radioprotection and Nuclear Safety, BP. 17, 92262 Fontenay-aux-Roses Cedex, France

\begin{abstract}
In the framework of criticality safety, the validation of the APOLLO2-MORET 4 calculation route, performed by IRSN, is based on the calculation of more than 1800 integral experiments. Calculations were performed on this huge validation database using group-wise nuclear data libraries based on JEFF-3.1 evaluation files. Calculation-experiment discrepancies were analyzed regarding the neutron spectrum. This validation highlights that the JEFF-3.1 evaluation gives markedly better results than those obtained with the JEF-2.2 evaluated library for many of the criticality applications.
\end{abstract}

\section{Introduction}

In the framework of the assessment of the criticality event, IRSN has been performed an extensive validation work of the APOLLO2-MORET 4 calculation route [1]. The validation database is composed of more than 1800 integral critical experiments mainly taken from ICSBEP Handbook [2]. Thus it spans a broad variety of configurations in terms of fissile medium, moderator, reflector and neutron spectrum.

Calculations were performed on this huge validation database using group-wise (172 groups) nuclear data libraries based on JEF-2.2 [3] and JEFF-3.1 [4] evaluation files. Calculation-experiment discrepancies are analyzed regarding the neutron spectrum focussing on areas for which JEFF-3.1 data improve significantly the prediction of the multiplication factor and on those for which further improvements to the nuclear data file should be sought for the benefits of criticality assessment.

\section{Calculations codes}

Calculations were performed with the APOLLO2-MORET 4 calculation route, which is well known as the standard route of the CRISTAL V1.1 french criticality package [5].

The APOLLO2 cell code [6] is used for self-shielding and for flux calculations with a 172 group-energy structure. Then, the obtained macroscopic cross sections are homogenized to be used in the MORET 4 Monte Carlo code [7] for 3D calculations with a general Pn-like anisotropy representation.

During the validation work, the use of point-wise Monte Carlo codes results (TRIPOLI4 and MCNP) enabled to emphasize code specific bias effects. The results presented in this paper intend to focus only on nuclear data effect.

To provide a strong test of the accuracy of the nuclear data, one must be confident about the quality of the integral experiments used in the validation process. Thus, different experimental sources were considered for each class of problem to avoid experimental programme specific tendencies. Finally, some of the experiments of the APOLLO2-MORET 4

${ }^{a}$ Presenting author, e-mail: isabelle.duhamel@irsn.fr validation database, which were proved to be not enough accurate for the nuclear data validation purpose [8], are not analysed in the present paper.

Finally, among the 1800 experiments of the APOLLO2MORET 4 validation database, about 1300 benchmarks were analysed to contribute to the JEFF-3.1 integral data testing.

Since the calculation-experiment discrepancies values, reported below as "C-E", have been obtained using the MORET 4 Monte Carlo code, a stochastic uncertainty has to be taken into account. Thus, the combined standard deviation associated to "C-E" value is given by the quadratic sum of the Monte Carlo calculation standard deviation and the $\mathrm{k}_{\mathrm{eff}}$ experimental uncertainty reported in the benchmarks. By the way, the Monte Carlo uncertainty was chosen to be rather small compared to the total experimental uncertainties.

Regarding the influence of the JEFF-3.1 evaluation file of the main isotopes, perturbation calculations were performed with the MORET 4 code based on the correlated sampling method to minimize the associated standard deviation.

\section{Benchmarking}

"C-E" values are analyzed regarding the neutron spectrum in order to highlight the main JEFF-3.1 improvements.

\subsection{Fast energy range}

Various plutonium and high-enriched uranium metallic systems were studied in order to evaluate the impact of the JEFF3.1 evaluation in the fast energy range.

Calculation-experiment comparisons for configurations involving bare assemblies allow focussing on the influence of the new JEFF-3.1 cross sections for ${ }^{239} \mathrm{Pu},{ }^{240} \mathrm{Pu}$, and ${ }^{235} \mathrm{U}$.

As shown in figure 1 , the new ${ }^{235} \mathrm{U}$ evaluation induces a small increase of the predicted $k_{\text {eff }}$ of about $0.15 \%$ in average in fast spectrum. Nevertheless, a systematic under-estimation still remains.

Regarding plutonium systems, the improvement in JEFF3.1 is really apparent, in particular for ${ }^{240} \mathrm{Pu}$, as shown for the benchmarks PMF002 and PMF029, which involve plutonium 


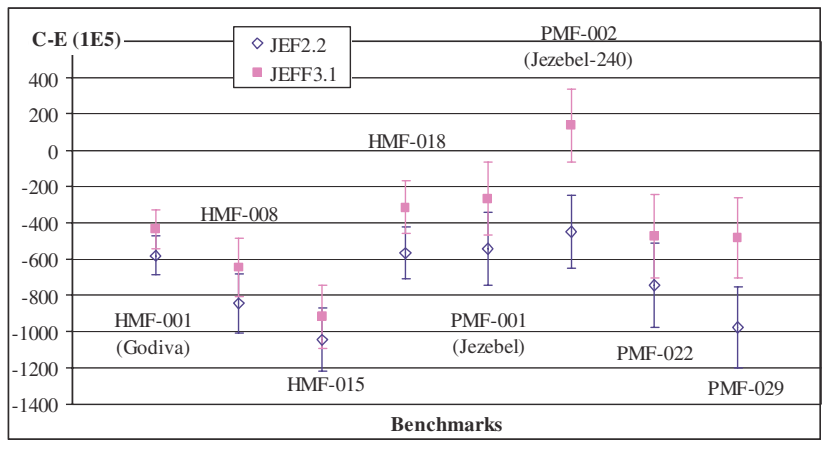

Fig. 1. Calculation-experiment comparisons using the JEF-2.2 and JEFF-3.1 evaluations for bare metallic systems.

metal with respectively $20 \%$ and $10 \%{ }^{240} \mathrm{Pu}$. In fact, the new evaluation allows reducing the under-prediction previously observed with JEF-2.2 cross sections. However, except for Jezebel-240 experiment for which JEFF-3.1 results are in good agreement with the experiment, a bias of $-0.4 \%$ in average still remains.

The Jezebel-U233 experiment, which involves a bare sphere of uranium metal with more than $98 \%{ }^{233} \mathrm{U}$, was also investigated. A $\mathrm{k}_{\mathrm{eff}}$ increase of about $1 \%$ was observed due to the $\mathrm{U}^{233}$ cross sections change in the fast energy range. This significant augmentation allows obtaining good agreement with the benchmark $\mathrm{k}_{\mathrm{eff}}$.

Once the actinides integral data testing performed in fast spectra, it is possible to evaluate the impact of JEFF-3.1 evaluation on the predicted $\mathrm{k}_{\mathrm{eff}}$ for structural materials in this energy range. Thus numerous configurations involving different reflectors with varying thickness were modelized and the corresponding "C-E" values were compared to those obtained with similar bare configurations.

A small decrease of the "C-E" values was observed for $\mathrm{CH}_{2}$ and water reflected configurations. Regarding graphite, a non-negligible impact was observed. The "C-E" values increase depending on the reflector thickness. Further analyses are in progress to study the angular distribution accuracy of these isotopes.

The new beryllium evaluation has been tested against 6 critical configurations. Contrary to JEF-2.2 evaluation, which leads to significant under estimated $\mathrm{k}_{\mathrm{eff}}$, the JEFF-3.1 library gives results within the uncertainties margins. Nevertheless, since bare metallic uranium systems were under predicted by about $0.5 \%$, it seems that inaccuracies still remain in beryllium nuclear data.

Four experiments involving spherical composite cores of plutonium and highly enriched uranium were also studied one bare configuration and three reflected by natural uranium. As discussed previously, the modifications of $\mathrm{Pu}$ and ${ }^{235} \mathrm{U}$ cross sections in JEFF-3.1 evaluation lead to a $\mathrm{k}_{\mathrm{eff}}$ increase depending on the ${ }^{240} \mathrm{Pu}$ content, which range from 2.3 to $16.1 \%$, and on the high enriched uranium thickness. Moreover, regarding natural uranium reflected experiments, the new ${ }^{238} \mathrm{U}$ nuclear data lead to a $\mathrm{k}_{\text {eff }}$ increase, which ranges from 0.49 to $0.67 \%$. The "C-E" values, which were previously under-estimated by $0.7 \%$ with JEF-2.2 libraries, are now within the experimental uncertainties. This might reflect the improvements on the ${ }^{238} \mathrm{U}$ angular distribution.

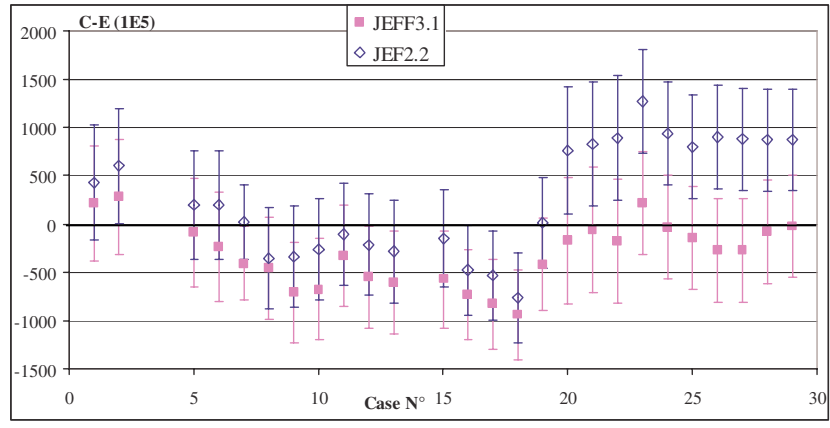

Fig. 2. Calculation-experiment comparisons using the JEF-2.2 and JEFF-3.1 evaluations for configurations with high-enriched uranium powders (HCM001).

\subsection{Intermediate energy range}

In order to contribute to ${ }^{235} \mathrm{U}$ nuclear data validation, highenriched uranium powders were investigated. These critical experiments consisted of cans in variously sized arrays containing enriched uranium dioxide $(93 \%)$ with different water content and various interspersed moderation configurations. A $15.24 \mathrm{~cm}$-thickness polyethylene reflector surrounded all experiments. Although categorized as "MIXED" in ICSBEP Handbook, some experiments also concern the fast and the thermal range.

As it can be observed on figure 2, the $\mathrm{k}_{\mathrm{eff}}$ decrease induced by the JEFF-3.1 evaluation allows obtaining a good agreement with the benchmark $\mathrm{k}_{\mathrm{eff}}$ for the configurations moderated by alcohol (cases 20 to 29), which were previously overestimated by $0.8 \%$.

Regarding the validation of ${ }^{238} \mathrm{U},{ }^{239} \mathrm{Pu}$ and ${ }^{240} \mathrm{Pu}$ in the intermediate energy range, the new experimental programme performed in BFS reactor by IPPE (Russia) has been investigated [9]. Most of these 11 experiments have been categorized as MIXED since there are less than $50 \%$ of fissions occurring in fast, intermediate and thermal range. APOLLO2-MORET 4 calculations results are presented in figure 3. Except for experiments with about $10 \%$ of ${ }^{240} \mathrm{Pu}$ (BFS-99), which were significantly over predicted, one can notice that JEFF3.1 leads to acceptable results. Five cases are within $1 \sigma$ and the two others within $2 \sigma$. Regarding the significant over predicted results

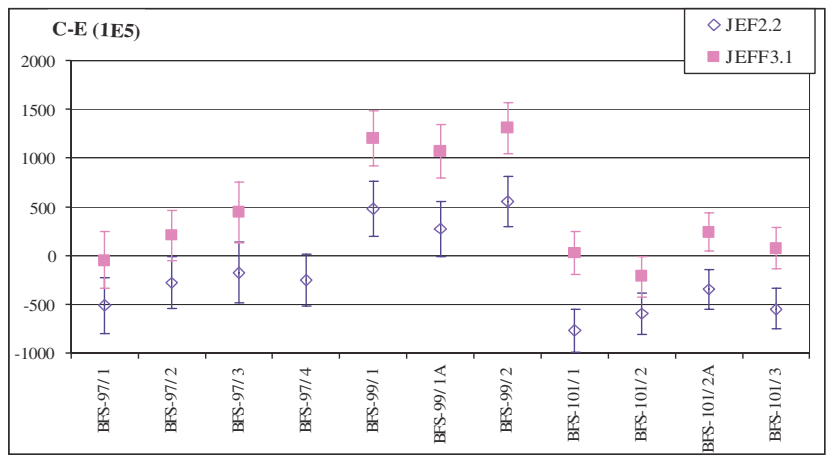

Fig. 3. Calculation-experiment comparisons using the JEF-2.2 and JEFF-3.1 evaluations for MMCM001 experiments. 


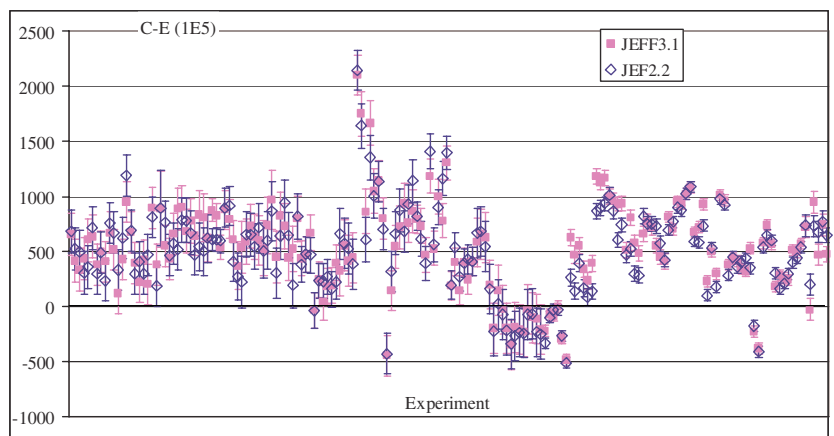

Fig. 4. Calculation-experiment comparisons using the JEF-2.2 and JEFF-3.1 evaluations for plutonium solutions.

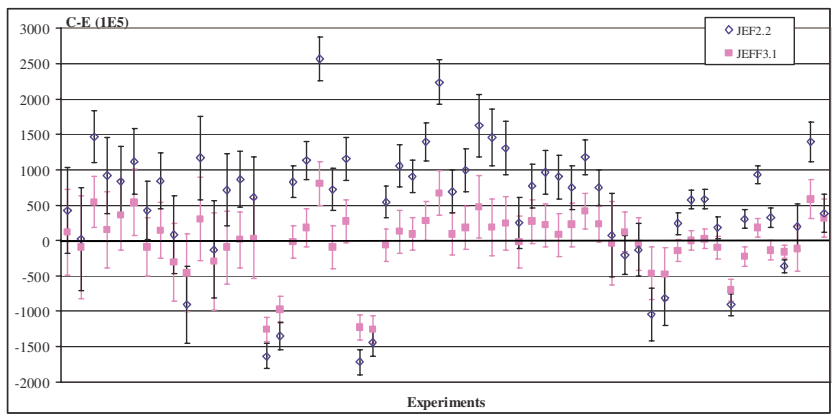

Fig. 5. Calculation-experiment comparisons using the JEF-2.2 and JEFF-3.1 evaluations for high-enriched uranium solutions.

for BFS-99, an experimental bias (on plutonium composition) is not excluded.

\subsection{Thermal energy range}

In order to contribute to the plutonium isotopes validation in thermal range, about 200 plutonium solution experiments were selected. They covered a broad range of plutonium concentration $(10 \mathrm{~g} / 1$ to $270 \mathrm{~g} / \mathrm{l})$ with various ${ }^{240} \mathrm{Pu}$ contents (0.5 to $30 \%)$.

Calculations results highlighted that the JEFF-3.1 evaluation does not allow reducing the over-estimation observed for the plutonium solutions, whatever the considered plutonium concentration. New improvements are still needed in the thermal spectra, especially for ${ }^{239} \mathrm{Pu}$ as already planned for JEFF-3.2 evaluation [10].

High-enriched uranium solutions with concentrations ranging from $15 \mathrm{~g} / \mathrm{l}$ to $730 \mathrm{~g} / \mathrm{l}$ were investigated to test the improvement of the ${ }^{235} \mathrm{U}$ cross sections in thermal and resonance range. Figure 5 shows the calculation-experiment discrepancies for both evaluations.

Contrary to calculations results obtained with JEF-2.2, which point out a general trend to $\mathrm{k}_{\text {eff }}$ over-prediction (about $0.9 \%$ in average), calculations with JEFF-3.1 give results in better agreement with the benchmark $\mathrm{k}_{\mathrm{eff}}$. This highlights the great improvement of the ${ }^{235} \mathrm{U}$ cross sections. The remaining discrepancies observed for some configurations allow questioning about the source biases. Further analyses are in progress to highlight experimental data bias or structural isotopes nuclear data inaccuracy.

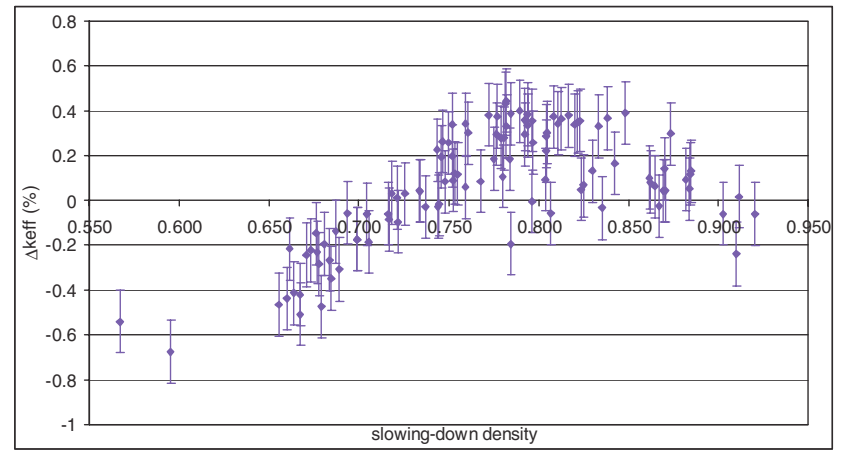

Fig. 6. Effect of nuclear data evaluation as a function of the slowing down density (ratio of fission neutrons slowing-down under $4 \mathrm{eV}$ ) for low-enriched $\mathrm{UO}^{2}$ rods lattices.

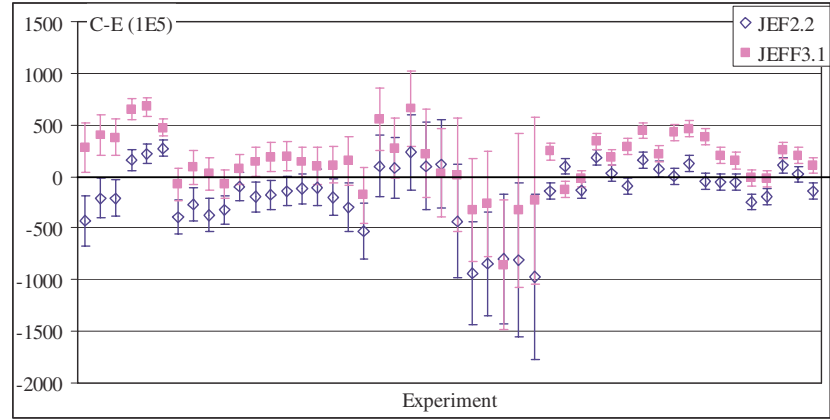

Fig. 7. Calculation-experiment comparisons using the JEF-2.2 and JEFF-3.1 evaluations for $\mathrm{UO}_{2}-\mathrm{PuO}_{2}$ lattices.

Experiments involving low-enriched uranium media were considered, contributing mainly to ${ }^{238} \mathrm{U}$ nuclear data validation in different spectra. The investigated experimental programmes involved low-enriched uranium solutions and uranium rods lattices.

Regarding uranium solutions with a concentration ranging from $170 \mathrm{~g} / \mathrm{l}$ to $980 \mathrm{~g} / \mathrm{l}$, a small decrease (about $0.15 \%$ on average) of the predicted $\mathrm{k}_{\mathrm{eff}}$ was observed when using the JEFF-3.1 evaluation, which allows reducing the small overprediction obtained with CRISTAL V1.1 standard route.

As for lattices, experiments involved arrays of lowenriched $\mathrm{UO}_{2}$ pins or uranium metal tubes. The uranium enrichment varies from $1.6 \%$ to about $7 \%$. Different square and triangular pitches of water-moderated arrays were considered in order to cover a wide range of moderation ratio.

Figure 6 presents the $\Delta \mathrm{k}_{\mathrm{eff}}$ (JEFF3.1-JEF2.2) variation versus the slowing-down density and highlights the impact of the new evaluation file depending on the neutron spectrum. A decrease of about $0.5 \%$, due to the new ${ }^{235} \mathrm{U}$ cross sections, is observed in the intermediate energy range, which leads to a reduction of calculation-experiment discrepancies when using JEFF-3.1 evaluation. On the contrary, for experiments with a more thermal spectrum, a small increase of $k_{\text {eff }}$ is noticed, which can sometimes lead to higher $\mathrm{C}-\mathrm{E}$ values than with JEF-2.2 evaluation. Nevertheless, they remain in the experimental uncertainties margins.

Arrays of $\mathrm{UO}_{2}-\mathrm{PuO}_{2}$ pins with various $\mathrm{PuO}_{2}(1 \%$ to $26 \%$ ) and ${ }^{240} \mathrm{Pu}(8 \%$ to $24 \%)$ contents and different square and triangular pitch lattices were also investigated. 
The impact of the JEFF-3.1 evaluation ranges from $-0.3 \%$ to $-0.7 \%$ depending not only on the plutonium content and the plutonium and uranium isotopic composition, but also on the moderation ratio. The observed over prediction confirms that improvements are still needed for plutonium cross sections.

A huge experimental programme [11] was conducted by IRSN in CEA Valduc (Apparatus B facility) to contribute to the validation of the six following fission products ${ }^{103} \mathrm{Rh},{ }^{133} \mathrm{Cs},{ }^{143} \mathrm{Nd},{ }^{149} \mathrm{Sm},{ }^{152} \mathrm{Sm}$ and ${ }^{155} \mathrm{Gd}$, as well as molybdenum, fluorine and natural gadolinium. Reference experiments (without the tested isotopes) has been also performed, thus they can be useful for the nuclear data testing. First calculations show a relative good agreement with the benchmark $\mathrm{k}_{\mathrm{eff}}$. However, possible inaccuracies on gadolinium, neodymium and rhodium are suspected. Sensitivity analyses are in progress to conclude on the C-E values observed.

\section{Conclusion}

The validation database of APOLLO2-MORET 4 standard route, which is composed of more than 1800 critical experiments, contains a broad variety of configurations in terms of fissile medium, moderator, reflector and neutron spectrum. Therefore, it has been used to contribute to the validation of the JEFF-3.1 nuclear data evaluation file.

Preliminary analyses have shown that JEFF-3.1 data improves significantly the calculation results in different areas. The $\mathrm{k}_{\text {eff }}$ prediction was markedly improved for high-enriched uranium systems in thermal and intermediate energy range thanks to the new ${ }^{235} \mathrm{U}$ evaluation. Moreover, the new ${ }^{238} \mathrm{U}$ data allow obtaining good results for water-moderated lowenriched uranium lattices and for fast systems reflected by depleted or natural uranium.

However, some improvements are still needed, especially for ${ }^{239} \mathrm{Pu}$ in thermal range and ${ }^{235} \mathrm{U}$ in fast energy range.

Regarding the on-going validation work, further analyses are in progress to analyse the structural isotopes impact in the different energy range.

Moreover, an important effort will be devoted to the integral data testing of fissions products.
The authors want to acknowledge CEA Saclay for providing the APOLLO2 libraries based on JEFF3.1, which were extensively used in this study.

\section{References}

1. I. Duhamel et al., The Experimental Validation of the APOLLO2MORET4 Standard Route of the French CRISTAL V1 Package, in Proceedings of the International Conference on Nuclear Criticality Safety, ICNC'2003, Tokai-Mura, Japan, Oct. 20-24, 2003.

2. International Handbook of Evaluated Criticality Safety Benchmark Experiments, Organization of Economic Cooperation and Development-Nuclear Energy Agency, NEA/NSC/DOC(95)03, September 2006 Edition.

3. The JEF-2.2 Data Library, JEFF Report 17, 2000.

4. The JEFF-3.1 Nuclear data Library, JEFF Report 21, NEA/OECD No 6190, 2006, ISBN 92-64-02314-3.

5. J.M. Gomit et al., CRISTAL V1: Criticality package for burn up credit calculations, in Proceedings of the International Conference on Nuclear Criticality Safety, ICNC'2003, Tokai-Mura, Japan, Oct. 20-24, 2003.

6. S. Loubière et al., APOLLO2 Twelve Years Later, in Proceedings of Mathematics and Computation, Reactor Physics and Environmental Analysis in Nuclear Applications, Madrid, Spain, 1999, Vol 2, pp. 1298-1315.

7. O. Jacquet et al., MORET: Version 4.B, a Multigroup Monte Carlo Criticality Code Package, in Proceedings of the International Conference on Nuclear Criticality Safety, ICNC'2003, Tokai Mura, Japan, Oct. 20-24, 2003.

8. I. Duhamel, S. Evo, Comparison results from APOLLO2MORET 4 validation database using JEF-2.2 and JEFF-3.1 nuclear data, in Proceedings of the International Conference on Nuclear Criticality Safety, ICNC'2007, St-Petersburg, Russia, May 28 - June 1, 2007 (to be published).

9. T. Ivanova et al., Impact of Nuclear Data on Multiplication Factor and Reactor Physics Parameters Calculation for Experiments Simulating Damp MOX Powders (these proceedings).

10. A.J. Koning et al., The JEFF evaluated nuclear data project (these proceedings).

11. N. Leclaire et al., Fission Products Experimental Programme, in Proceedings of the International Conference on reactor physics, PHYSOR 2006, Vancouver, Canada, Sept. 10-14, 2006. 